\title{
(2) MAVRICS: A Robust and Safe Magnetic Nanoparticle based RNA Extraction Method Compatible with Phenol- chloroform Inactivated Infectious Samples V.3
}

Mo Li ${ }^{1}$, Gerardo Ramos-Mandujano ${ }^{2}$

${ }^{1}$ King Abdullah University of Science and Technology;

${ }^{2}$ Laboratory of Stem Cell and Regeneration, Biological and Environmental Science and Engineering Division, King Abdullah Uni versity of Science and Technology (KAUST)

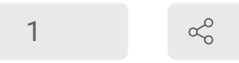

dx.doi.org/10.17504/protocols.io.b2p8qdrw

Mo Li

King Abdullah University of Science and Technology

Diagnosis and surveillance of emerging pathogens such as SARS-CoV-2 depend on nucleic acid isolation from clinical and environmental samples. Under normal circumstances, samples would be processed using commercial proprietary reagents in Biosafety 2 (BSL-2) or higher facilities. A pandemic at the scale of COVID-19 has caused a global shortage of proprietary reagents and BSL-2 laboratories to safely perform testing. Therefore, alternative solutions are urgently needed to address these challenges. We developed an opensource method called Magneticnanoparticle-Aided Viral RNA Isolation of Contagious Samples (MAVRICS) that is built upon reagents that are either readily available or can be synthesized in any molecular biology laboratory with basic equipment. Unlike conventional methods, MAVRICS works directly in samples inactivated in acid guanidinium thiocyanate-phenol-chloroform (e.g., TRIzol), thus allowing infectious samples to be handled safely without biocontainment facilities.

DOI

dx.doi.org/10.17504/protocols.io.b2p8qdrw

https://www.medrxiv.org/content/10.1101/2020.06.28.20141945v1

Mo Li, Gerardo Ramos-Mandujano 2021. MAVRICS: A Robust and Safe Magnetic Nanoparticle based RNA Extraction Method Compatible with Phenol-chloroform Inactivated Infectious Samples. protocols.io https://dx.doi.org/10.17504/protocols.io.b2p8qdrw

Mo Li

KAUST

Grant ID: BAS/1/1080-01

SARS-CoV-2, MAVRICS, COVID-19, RNA extraction, Magnetic nanoparticle synthesis, Silica magnetic nanoparticles (SiMNP)

protocol ,

Dec 08, 2021

Dec 08, 2021

55776

Citation: Mo Li, Gerardo Ramos-Mandujano MAVRICS: A Robust and Safe Magnetic Nanoparticle based RNA Extraction Method Compatible with Phenol-chloroform Inactivated Infectious Samples https://dx.doi.org/10.17504/protocols.io.b2p8qdrw

This is an open access protocol distributed under the terms of the Creative Commons Attribution License (https://creativecommons.org/licenses/by/4.0/), which permits unrestricted use, distribution, and reproduction in any medium, 


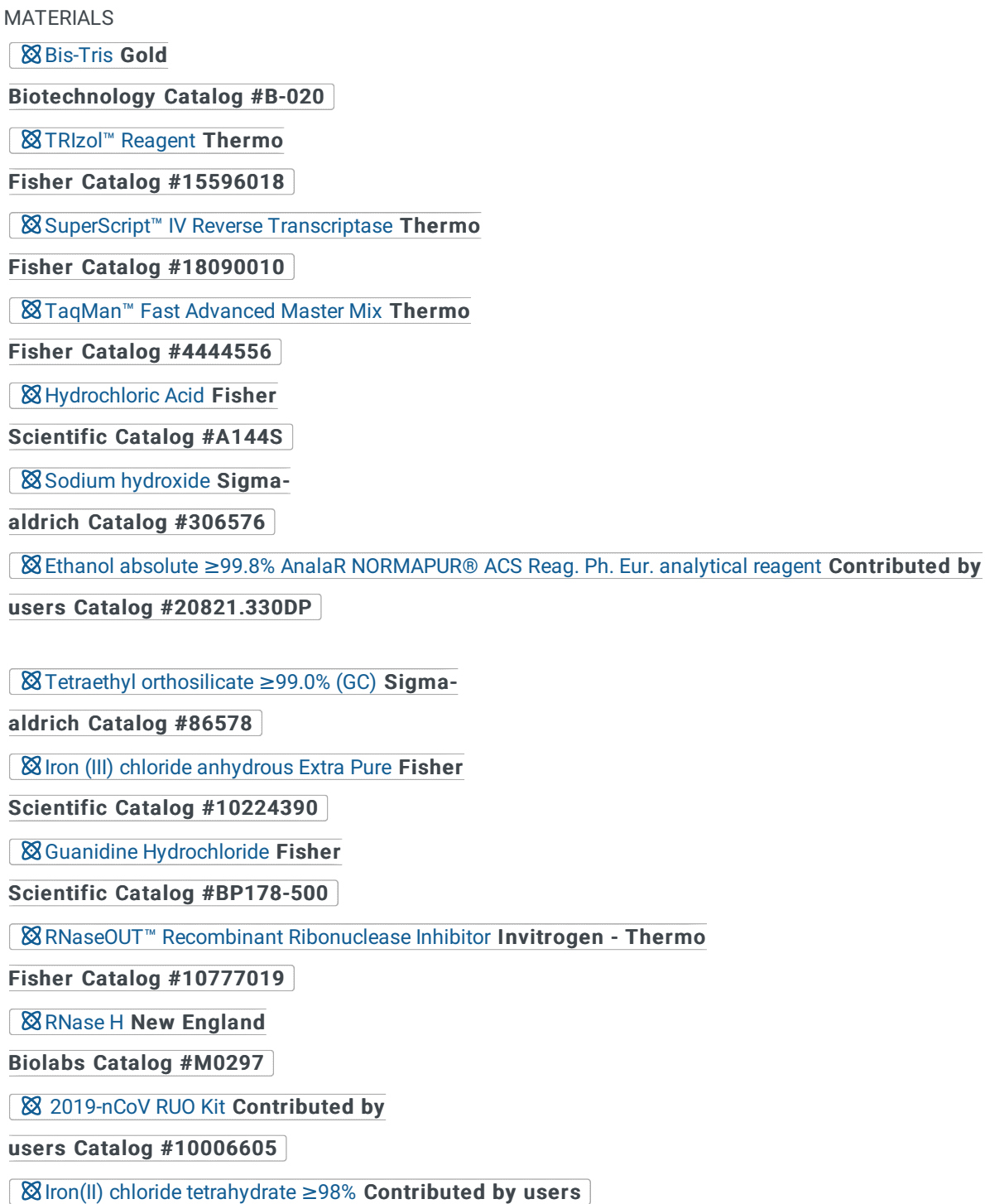

Q Iron(II) chloride tetrahydrate $\geq 98 \%$ Contributed by users

1 Silica magnetic nanoparticles (SiMNP) synthesis.

SiMNP synthesis was done following the published protocols in BOMB.bio: BOMB magnetic core nanoparticles synthesis and BOMB coating ferrite MNPs with silica oxide.

@ Overnight

\section{2 (11)}

COVID-19 patient samples.

Oropharyngeal or nasopharyngeal swabs are steeped in $\boxminus 1 \mathrm{~mL}$ acid guanidinium thiocyanate-phenol-chloroform (AGPC, e.g., TRIzol Reagent or TRI reagent).

A Follow CDC or institutional safety guidlines when handling potential infectious samples. AGPC, TRIzol and TRI reagent contain phenol. Follow local safety guidelines when handling and disposing these reagents.

仓0:01:00

\section{Making Bis-Tris Buffer $\square 50 \mathrm{~mL}$}

\section{(6) protocols.io}

Citation: Mo Li, Gerardo Ramos-Mandujano MAVRICS: A Robust and Safe Magnetic Nanoparticle based RNA Extraction Method Compatible with Phenol-chloroform Inactivated Infectious Samples https://dx.doi.org/10.17504/protocols.io.b2p8qdrw

This is an open access protocol distributed under the terms of the Creative Commons Attribution License (https://creativecommons.org/licenses/by/4.0/) which permits unrestricted use, distribution, and reproduction in any medium, 
3.1 Dissolve $\boxminus \mathbf{1 4 . 3 3} \mathbf{g}$ guanidinium hydrochloride and $\square \mathbf{1 0 4 . 6} \mathbf{~ m g ~ B i s - T r i s ~ i n ~} \square \mathbf{5 0} \mathrm{mL}$ of $90 \%$ ethanol. $@ 00: 10: 00$

If Bis-Tris is not available, it may be substituted by Tris Base ( $10 \mathrm{mM}$ final concentration)

Add $90 \%$ ethanol to the chemicals to a final volume of $45-48 \mathrm{ml}$ and wait for guanidinium hydrochloride to completely dissolve by mixing.

3.2 Adjust $\mathrm{pH}(<6.5)$ with $\mathrm{HCl}$, and adjust the volume with $90 \%$ of ethanol to $\square \mathbf{5 0} \mathbf{~ m L}$.

@00:05:00

4 Magnetic-nanoparticle-Aided Viral RNA Isolation of Contagious Samples.

\section{$4.1 \triangle$}

In an Eppendorf tube add $\square \mathbf{2 0 0} \mu \mathrm{l}$ clinical sample, $\boxminus \mathbf{2 0 0} \mu \mathrm{l}$ Bis-Tris buffer, mix well by vortexing. $\bullet 00: 01: 00$

We recommend Lo-Bind Eppendorf tubes or similar low binding tubes.

4

Samples contain phenol. Cap the tubes slowly. Make sure the tubes can be closed securely during vortexing.

4.2 Add $\boxminus 40 \mu \mathrm{l}$ SiMNP, mix at $\boxminus 1300 \mathrm{rpm} \circlearrowleft 00: 05: 00$

The SiMNP stock is further diluted with RNase-free water to be used for RNA extraction. Recommended dilution for swab samples mixed with TRIzol is 1 volume of fully resuspended SiMNP stock to 4 volumes of RNase-free water. The optimal ratio for other samples should be determined empirically; typical dilution ranges from 1:4 to 1:10.

4.3 Spin the tube for 2-3 seconds, settle the SiMNPs on a magnetic stand and remove the supernatant. 00:02:00

The supernatant contains phenol. Follow local safety guidelines when handling and disposing these reagents.

Citation: Mo Li, Gerardo Ramos-Mandujano MAVRICS: A Robust and Safe Magnetic Nanoparticle based RNA Extraction Method Compatible with Phenol-chloroform Inactivated Infectious Samples https://dx.doi.org/10.17504/protocols.io.b2p8qdrw

This is an open access protocol distributed under the terms of the Creative Commons Attribution License (https://creativecommons.org/licenses/by/4.0/) which permits unrestricted use, distribution, and reproduction in any medium, 


\section{$4.4 \%$}

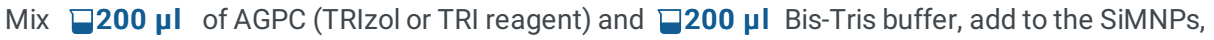
mix well by vortexing. $\bullet$ 00:01:00

\section{$4.5 \mathscr{0}$}

Settle the SiMNPs on a magnetic stand and remove the supernatant. @ 00:02:00

1. The supernantant contains phenol. Follow local safety guidelines when handling and disposing these reagents.

\section{$4.6 \not 0$}

Add $\boxminus \mathbf{4 0 0} \mu \mathrm{l}$ of $90 \%$ ethanol and mix well, spin for $2-3$ seconds, settle the siMNPs on a magnetic stand and remove the supernatant. $\bullet 00: 02: 00$

It is highly recommended to prepare fresh $90 \%$ ethanol before use. Make sure the $90 \%$ ethanol container is closed tightly to prevent evaporation.

\section{$4.7 \mathscr{0}$}

Repeat Setp 4.6 three more times for a total of 4 ethanol washes $\bullet$ 00:06:00

\section{8 个}

After removing the supernatant from the last ethanol wash, dry the SiMNPs on a heat block at $50^{\circ} \mathrm{C}$. Keep the lid open, no shaking. Do not elute before the SiMNPs are dried. $\bullet$ 00:20:00

Drying may take less than 20 min. Monitor the appearance of the SiMNPs during drying. The SiMNPs appear in a rusty brownish color when dried.

4.9 To elute the RNA, add $\boxminus \mathbf{4 0} \boldsymbol{\mu l}$ nuclease-free water, and mix at $\boxminus \mathbf{1 3 0 0} \mathrm{rpm}$ for $\circlearrowleft$ 00:05:00 at room temperature.

\subsection{0 全}

Settle the SiMNPs on a magnetic stand and transfer the eluted RNA to a new RNase-free tube.

\section{(6) protocols.io}

Citation: Mo Li, Gerardo Ramos-Mandujano MAVRICS: A Robust and Safe Magnetic Nanoparticle based RNA Extraction Method Compatible with Phenol-chloroform Inactivated Infectious Samples https://dx.doi.org/10.17504/protocols.io.b2p8qdrw

This is an open access protocol distributed under the terms of the Creative Commons Attribution License (https://creativecommons.org/licenses/by/4.0/) which permits unrestricted use, distribution, and reproduction in any medium, 
৫0:02:00

Pipet slowly, avoid taking up SiMNPs.

\section{$4.11 \circledast$}

Analyze RNA concentration and purity using a Qubit fluorometer or Nanodrop. @ 00:10:00

4.12 Store RNA at $-80^{\circ} \mathrm{C}$ or use immediately.

5 Reverse transcription (RT): use $\boxminus \mathbf{4} \mu \mathrm{l}$ of eluted RNA and follow the instructions for SuperScript ${ }^{\mathrm{TM}}$ IV Reverse Transcriptase adding the RNase $\mathrm{H}$ incubation step. @ 01:00:00

User should optimize the input RNA amount.

$6 \not{\nabla}$

Real-time PCR: For each $\square \mathbf{1 0} \boldsymbol{\mu l}$ qPCR reaction mix $\square \mathbf{1 . 5} \boldsymbol{\mu l}$ cDNA, $\square \mathbf{0 . 5} \boldsymbol{\mu l}$ SARS-CoV-2 (2019-nCoV) CDC qPCR Probe Assay, $\square \mathbf{5} \boldsymbol{\mu l}$ TaqMan Fast Advanced Master Mix, and $\boxminus \mathbf{1 . 5} \boldsymbol{\mu l}$ nuclease-free water. Run qPCR on a Biorad CFX384 Touch Real-Time PCR Detection System (or similar instrument) using the following program: $50^{\circ} \mathrm{C}$ for $2 \mathrm{~min}, 95^{\circ} \mathrm{C}$ for $2 \mathrm{~min}$ followed by 45 cycles of $95^{\circ} \mathrm{C}$ for $5 \mathrm{sec}$ and $59^{\circ} \mathrm{C}$ for $30 \mathrm{sec}$. $01: 20: 00$

Citation: Mo Li, Gerardo Ramos-Mandujano MAVRICS: A Robust and Safe Magnetic Nanoparticle based RNA Extraction Method Compatible with Phenol-chloroform Inactivated Infectious Samples https://dx.doi.org/10.17504/protocols.io.b2p8qdrw

This is an open access protocol distributed under the terms of the Creative Commons Attribution License (https://creativecommons.org/licenses/by/4.0/), which permits unrestricted use, distribution, and reproduction in any medium, 